\title{
Curbing the Opioid Epidemic at its Root: The Effect of Provider Discordance after Opioid Initiation
}

\begin{abstract}
While medical research has addressed the clinical management of chronic opioid users, little is known about how operational interventions shortly after opioid initiation can impact a patient's likelihood of long-term opioid use. Using a nationwide US database of medical and pharmaceutical claims, we investigate the care delivery process at the most common entry point to opioid use: the primary care setting. For patients who return to primary care for a follow-up appointment within 30 days of opioid initiation, we ask: who should revisit (and potentially revise) the opioid-based treatment plan, the initial prescriber (provider concordance) or an alternate clinician (provider discordance)? First, using a fully controlled logistic model, we find that provider discordance reduces the likelihood of long-term opioid use 12 months after opioid initiation by $31 \%$ (95\% CI: $[18 \%, 43 \%])$. Both an instrumental variable analysis and propensity-score matching (utilizing the minimum-bias estimator approach) that account for omitted variable bias indicate this is a conservative estimate of the true causal effect. Second, looking at patient activities immediately after the follow-up appointment, we find that this long-term reduction is at least partially explained by an immediate reduction in opioids prescribed after the follow-up appointment. Third, the data suggest that the benefit associated with provider discordance remains significant regardless of whether the patient's initial prescriber was their regular provider (versus another clinician). Overall, our analysis indicates that systematic, operational changes in the early stages of managing new opioid patients may offer a promising, and hitherto overlooked, opportunity to curb the opioid epidemic.
\end{abstract}

\section{INTRODUCTION}

Over the past 30 years, the opioid epidemic has escalated to claim more than 130 lives each day in the United States. Opioid overdoses resulted in 47,600 deaths in 2017 alone, a 12\% increase from 2016 (Scholl et al. 2019), rendering the epidemic one of the driving contributors to the nation's recent decrease in life expectancy (Dowell et al. 2017). To curb inappropriate opioid usage, the US has introduced interventions including prescription drug monitoring programs, increasing the availability of overdose-preventing drugs, and funding research into abusedeterrent or tamper-resistant opioid formulations (Grosser et al. 2017, Pitt et al. 2018). However, as demonstrated by the surge in opioid-related overdoses and deaths during the recent Covid-19 crisis (American Medical Association 2020, Holland et al. 2021), the opioid epidemic is far from over.

One limitation of many current opioid-reducing initiatives is their focus on high-risk chronic opioid users patients filling high dosage opioid prescriptions or heroin users. These high-risk patients can be difficult to treat, as they are often dependent on prescribed opioids or else obtain opioids illicitly and no longer rely on the healthcare system to provide opioid access (Pitt et al. 2018, Scholl et al. 2019). Moreover, while these patients are at the highest immediate risk of overdose, they are, in a sense, only the tip of the iceberg. With the majority of heroin users reporting that their journey to dependence began with a legal opioid prescription (Compton et al. 2016), more research is needed to identify early interventions that could effectively disrupt the pathway from first prescription to opioid dependence. 
We address this gap in the literature by empirically examining the process of managing new opioid initiates in the primary care setting. Primary care clinicians are the largest group of opioid prescribers and often serve as the first encounter for patients with noncancer pain (Grosser et al. 2017, Levy et al. 2015). While some patients do not return to the primary care setting after their initial opioid prescription (either because their pain has subsided or because they receive subsequent care from a different specialty), other patients return for further diagnosis or to assess the progress of treatment. This follow-up appointment offers a chance to revisit and potentially revise the initial treatment plan based on health progression and any other new information.

For those patients who continue seeking treatment in the primary care setting after opioid initiation, we therefore ask: who should revisit the treatment plan with the patient, the original prescriber (i.e., provider concordance) or another clinician (i.e., provider discordance)? ${ }^{1}$ While a different clinician can expose the patient to a "fresh perspective" and prevent anchoring to the original opioid treatment course, it may also lead to more fragmented care and reduce physician "ownership" of the long-term health of the patient (Ahuja et al. 2020, Ariely et al. 2003, Senot 2019). Although prescriber continuity is typically recommended for patients already dependent on opioids (Hallvik et al. 2018, Jena et al. 2014), the overall impact of exposing a patient to variation in providers in the initial stages of opioid use is not immediately clear.

We examine the care delivery process for new opioid initiates in the primary care setting by leveraging a nationwide claims database of more than 3.5 million patients. First, we find empirical evidence that incorporating provider discordance early in the care management process may reduce long-term opioid use rates by at least $31 \%$ (95\% confidence interval [CI]: 18\%, 43\%). After identifying the main effect, we analyze a potential mechanism for the relationship: whether the patient fills a subsequent opioid prescription after the follow-up appointment. We also examine different discordant care pathways to determine whether the effect size differs depending on whether the patient was prescribed opioids by their regular (i.e., most frequently visited) primary care provider or by another clinician.

While continued opioid use is clinically justified and effectively reduces pain for some patients, the risks of inappropriate or long-term opioid use can be severe (Dowell et al. 2017, Glod 2017, Pitt et al. 2018). Overall, this research suggests that investigation into systematic changes following the initiation of opioids could be a promising and hitherto overlooked opportunity to reduce the influx of patients afflicted by the opioid epidemic.

\section{THEORY AND HYPOTHESIS DEVELOPMENT}

\subsection{Related Literature}

In the early stages of opioid use, it is not always clear whether the pain relief offered by opioid treatment will outweigh the possible risks of clinical harm and dependence (Deyo et al. 2017, Dowell et al. 2016). This uncertainty can lead to variation in care, such that a patient may receive a different treatment based on nonclinical factors, including the medical knowledge of the diagnosing clinician and location of care (Green 2012). This phenomenon has been acutely observed in the opioid context, where the number of opioid pills consumed

\footnotetext{
${ }^{1}$ Note we do not make any evaluation on the clinical efficacy of the opioid regimen. Instead, we explore a change to the management process (i.e. provider discordance or concordance after opioid initiation) that does not directly impact clinical judgement but may materially impact the patient's likelihood of long-term opioid use.
} 
by patients after surgery varied markedly depending on, e.g., the default opioid pill count presented in the electronic medical record system and the quantity contained in a first prescription (Chiu et al. 2018, Farley et al. 2019).

Traditionally, the healthcare operations community has helped address care variation by developing decision support tools that, e.g., prioritize hospital discharge decisions (Chan et al. 2012), optimize cancer screening schedules (Ayer et al. 2012), inform hospital capacity decisions (Song et al. 2019), and balance fairness with efficiency when allocating organ transplant resources (Dickerson et al. 2019). In their respective clinical contexts, these tools effectively minimize unwarranted care variation and improve overall patient outcomes. However, in the context of opioids where clinicians lack an objective scale to measure the patient's level of pain (Morone and Weiner 2013), algorithms have limited utility in reducing variation of care.

Meanwhile, researchers have recently started to explore the potential benefits of variation when introduced within the care pathway of an individual patient (Ahuja et al. 2020, Chan et al. 2019, Kuntz et al. 2019). Especially for medical conditions where multiple viable treatment options exist, patients may benefit from exposure to a variety of providers and treatment options as they search for the best solution to their problems (Christensen et al. 2009). For pain management in particular, pharmacological therapy such as opioids represents only one of many potential treatment modalities; alternatives include physical rehabilitation, psychological therapy, and interventional treatments such as injections and surgical procedures (Owen et al. 2018).

In the early stages of pain management, the question of which provider is best positioned to revisit the treatment plan is akin to the notion of gatekeeping. Past research has investigated the initial provider, who decides whether to manage the patient's treatment alone or to refer the patient to a specialist (Freeman et al. 2017, 2020, Shumsky and Pinker 2003). In these settings, the additional cost of treatment by a specialist is weighed against the cost of failing to solve the patient's problem without specialist input. Our context also involves the patient potentially seeing two different clinicians. However, while the gatekeeping notion is predicated on referral to a specialist, we focus on alternative providers within the same care level (i.e., primary care). Furthermore, gatekeeping is an active, endogenous escalation decision by the initial clinician; in contrast, we study an exogenous process change (e.g., to patient scheduling) that is not necessarily taken by the initial prescriber.

This concept of routing during the early stages of opioid use is also related to studies on continuity of care. Such work typically focuses on the long-term management of patients who are already suffering from specific chronic conditions, e.g., heart failure (Senot 2019), diabetes (Ahuja et al. 2020), or opioid dependence (Hallvik et al. 2018). While most studies in these chronic settings find that patients benefit from repeated appointments with the same practitioner, we focus on patients prior to chronicity. Additionally, we highlight the importance of earlystage management as part of a strategy to prevent risk escalation in the first place. As outlined below, we hypothesize that the process of care delivery (namely provider concordance or discordance) during these critical initial stages may have a significant bearing on later outcomes.

\subsection{The Effect of Provider Discordance}

In medical settings, clinician discordance is generally recognized to confer two main advantages. First, combining the knowledge pools of two clinicians enlarges the information base used in decision-making (Ahuja et al. 2020, 
Brooks et al. 2015). The new clinician may know of alternative treatments or could elicit new information from the patient that proves important in weighing the trade-offs between potential treatments. This new perspective may be particularly important for the early stages of opioid use given the array of clinically viable treatment options available for pain management (Owen et al. 2018).

Second, the sequential, independent decision-making process involved when seeing two different clinicians can help counteract cognitive biases such as confirmation bias and anchoring (Ariely et al. 2003, Gino and Pisano 2008). Following the initial decision to prescribe an opioid, a practitioner's desire to remain coherent over time may bias them against stopping the prescription. For example, Staats et al. (2018) show that providers with more experience may get caught in the status quo, rendering them less able to objectively update their beliefs in light of new information. A change in provider may thus increase the odds of a patient being transitioned to a new (i.e., non-opioid) course of treatment.

Despite potential benefits, clinician discordance is typically discouraged in medicine as it increases fragmentation of care. Receiving care from multiple clinicians can result in lapses in communication and coordination, exacerbated when providers do not share clinical data (Clark et al. 2013, Senot 2019). Both tacit and explicit information can be lost, reducing providers' opportunities to learn (e.g., by augmenting existing data with new information) and to adjust treatment over time (Reagans et al. 2005).

With respect to opioid-management specifically, the medical literature has examined the role of provider discordance in the post-acute phase of opioid-use. Since the past prescription is a known risk factor for longterm use (Deyo et al. 2017), a provider's awareness of the patient's opioid history may reduce their likelihood of offering additional opioids. Studies have also identified that involving fewer distinct clinicians in chronic opioid management is associated with a reduced number of high-risk prescriptions, opioid-related hospitalizations, and overdose (Hallvik et al. 2018, Jena et al. 2014).

Generally, in this post-acute phase of opioid use, it appears important for patients to avoid the potential disadvantages of care fragmentation. However, to our knowledge, the value of seeing multiple primary care clinicians during the initial phase of opioid use has not been evaluated. We believe that for these new opioid users, the advantages of clinician discordance (an enlarged information base and correction to confirmation bias and anchoring) are likely to outweigh the disadvantages. This is captured in Hypothesis 1:

Hypothesis 1. After an initial opioid prescription, if the patient's follow-up appointment is with an alternate clinician compared to their initial opioid prescriber (provider discordance), then the patient's likelihood of becoming a long-term opioid user is reduced.

\subsection{A Potential Mechanism}

If a follow-up appointment with an alternate clinician reduces the likelihood of becoming a long-term opioid user (Hypothesis 1), then one would also expect a change in the patient's short-term opioid usage. A natural candidate for a mechanism in the relationship between provider discordance and long-term opioid use is the patient's opioid use shortly after the follow-up visit. 
If this relationship exists, we expect to observe that patients who see a different doctor for their follow-up appointment will be less likely to fill an opioid prescription soon after the follow-up visit, following the arguments of Section 2.2. We also expect to observe an association between filling an opioid prescription soon after the follow-up appointment and the rate of long-term opioid use. These associations are tested in Hypotheses 2a-b:

Hypothesis 2a. Patients who experience provider discordance are less likely to fill an opioid prescription immediately after the follow-up appointment.

Hypothesis 2b. Patients who fill an opioid prescription immediately after their follow-up appointment are more likely to become long-term opioid users.

Note that our intention is not to establish a causal connection through these hypotheses. Instead, we aim to provide further evidence of the effect of provider discordance on long-term opioid use (Hypothesis 1) by investigating an observable change in patient activity shortly after the follow-up appointment.

\subsection{Provider Discordance Pathways}

Many patients have a regular primary care provider who the patient sees most frequently for their health needs (Atlas et al. 2009). Through repeated interactions, the patient's regular provider typically will have acquired knowledge of the patient's comorbidities, medical, and family history. Likewise, a patient is more likely to have familiarity and trust in their regular provider, creating an environment where they may be more willing to share personal information (Siemsen et al. 2009).

As a regular provider is more likely to take personal responsibility or ownership for the patient's long-term health (Jena et al. 2014, Senot 2019), we anticipate that they are well-positioned to balance short-term pain against longterm risks of continued opioid use. Therefore, we would expect a regular provider to be more likely than an alternate clinician to encourage conservative treatment plans. Likewise, greater familiarity and trust may increase a patients' willingness to explore alternative non-opioid treatment options proposed by a regular provider (Ahuja et al. 2020b).

Not only could seeing a regular provider have a net impact on opioid use, but patients who switch to and from their regular clinician in the early stages of opioid use may not benefit equally from provider discordance. For instance, we might expect that patients who were initially prescribed opioids by their regular provider will receive little benefit from discordance if the follow-up provider is less willing to adjust the regular provider's care plan. By comparison, a patient who initiates opioids with an alternate provider (perhaps due to acute onset of pain and unavailability of their regular provider) then switches to their regular provider for follow-up may benefit greatly from the fresh perspective and familiarity of their regular provider. This motivates Hypothesis 3 :

Hypothesis 3. The relative reduction of long-term opioid use rates from provider discordance is stronger when patients change from an alternate clinician to their regular provider for the follow-up appointment, compared to patients who switch away from their regular provider to an alternate clinician. 


\section{DATA SOURCES AND VARIABLE CONSTRUCTION}

\subsection{Data Description}

For this retrospective cohort study, we used a multi-payer dataset that includes the insurance claim information of approximately 3.5 million beneficiaries in the US. While claims data are complex to analyze - often containing duplicated or missing information - they are also a rich source for observational studies (Hopp et al. 2018, Jena et al. 2014). They contain past utilization patterns, information about patient comorbidities, prescription fills, and details of the clinicians involved in the patient journey.

Patients in the sample were insured by a commercial or government (Medicare or Medicaid) plan and each has at least 18 months of continuous medical and pharmaceutical claims eligibility between September 2012 and December 2018. This continuous eligibility requirement ensured that we could observe the patient history both before and after their initial opioid prescription. The patient-level eligibility data also included the date of birth, gender, and location information for each unique patient-payer entry.

The medical claims data contain information including the unique patient and payer identifier, up to the first ten diagnosis codes (International Classification of Disease, ICD9 and ICD10), rendering provider identifier (linked to the National Provider Identifier [NPI]), and service location type (office, inpatient, emergency department, etc.). The NPI was linked to the National Plan and Provider Enumeration System (NPPES) database to obtain clinician information including credentials (MD, DO, PA, NP, etc.), specialty description, and office name and location(s).

The pharmaceutical claims data contain information including the unique patient identifier, prescribing physician identifier (linked to the NPI), generic product identifier (GPI) classification, drug base, drug strength, drug dosage form (e.g., tablet, patch), prescription quantity, days supplied, and filled date. The research methodology was reviewed and approved by the Ethics Committee at [INTENTIONALLY LEFT BLANK].

\subsection{Sample Selection}

To ensure clinical accuracy and interpretability, we closely followed related medical literature on opioid misuse when selecting the sample (Barnett et al. 2017, Dowell et al. 2016, Jena et al. 2014). New opioid users were identified using the two-digit GPI classification in the pharmaceutical claims. After a clean period of at least six months during which no opioid prescriptions were filled, we identified the start of a new opioid episode based on when the patient filled their index opioid prescription.

We then used the prescriber's unique identifier to subset the sample to new patient opioid use episodes that could be linked to a corresponding medical claim (considered to be the prescribing visit) in the 14 days prior to filling the prescription (Hoopes et al. 2018). The prescribing appointment must have taken place in the primary care office setting, which we identified using the Centers for Medicare and Medicaid Services (CMS) service location code and the prescriber's specialty as reported in NPPES.

To study the beginning phase of primary care management for new opioid initiates, we excluded all patients who did not return to the primary care setting within 30 days of filling the initial opioid prescription for the same 
condition. Consistent with defining the study sample, any corresponding intervention should be designed only to target patients who were already returning to the primary care for follow-up (i.e., not the "one-and-done" patients). However, for completeness, models within Supplement Methods S14 account for this censoring to estimate the effect of provider discordance if the intervention were applied to the full population of opioid initiates.

We also excluded patients who were diagnosed with cancer or end-stage renal disease or who were under the age of 18 at the start of the episode. In this way, we identified 11,340 new opioid patients who had follow-up appointments within 30 days. Supplement Part I provides summary statistics of the patient sample and a detailed list of inclusion and exclusion criteria.

\subsection{Independent Variable}

For each opioid episode, we identified the patient's first follow-up appointment in the primary care setting occurring within 30 days after filling the initial opioid prescription. To increase confidence that the follow-up was related to the initial prescription, the prescribing and follow-up appointments were required to share at least one of the top three ICD diagnosis chapters. A binary independent variable captured whether the initial prescriber was different from the clinician seen for the follow-up appointment (i.e., provider discordance). Of the 11,340 episodes in the sample, $3,211(28.3 \%)$ experienced provider discordance.

\subsection{Dependent Variable}

The primary dependent variable was long-term opioid use. This is typically defined in the medical literature as the patient having filled a minimum of 180 days supplied of opioid prescriptions within the first 360 days after opioid initiation, excluding any days supplied within the first 30 days of the initial prescription (Barnett et al. 2017). The 30-day exclusion period separates the exposure period from the outcome measure, eliminating the concern that the exposure and outcome are correlated by definition.

Because the days supplied field is often auto-populated in pharmaceutical claims, we confirmed robustness of the findings by computing an alternative definition of long-term opioid use that does not depend on the days supplied. Using the CMS standard baseline conversion to compute the full prescription dosage, we calculated patients' daily strength of opioids using the number of days between prescription filled dates (Centers for Medicare and Medicaid Services 2018). Patients were flagged for long-term opioid use if they had a daily dosage of $20 \mathrm{MME}$ or higher 360 days after the initial prescription, a moderate threshold associated with an elevated risk of opioid-related complications and overdose (Dowell et al. 2016).

The two measures of long-term opioid use will be referred to as the days supplied or daily strength definitions throughout the paper. Whenever the measurement is not specified in the paper, the results correspond to the days supplied measure. In our sample, $10.0 \%$ of patients were considered long-term opioid users based on the days supplied definition and 5.9\% using the daily strength definition. The rates of long-term opioid use were higher amongst patients who experienced provider concordance ( $11.5 \%$ and $6.9 \%$, using the two long-term opioid use definitions, respectively) compared to those who experienced provider discordance $(6.2 \%$ and $3.4 \%$, 
respectively). More information on the construction of the dependent variable can be found in Supplement Methods S2.

\subsection{Controls}

To isolate the impact of provider discordance on long-term opioid use, it is important to control for any observable factors which influence both provider discordance and a patient's propensity to continue opioid use. First, we gathered characteristics of the initial prescription which may be related to the patient's acuity (i.e., the speed of onset of the condition and need for timely evaluation) and severity (i.e., how bad the condition is). These included covariates for the drug base, average daily opioid strength, and prescription days supplied of the initial opioid prescription (Deyo et al. 2017, Levy et al. 2015). ${ }^{2}$ We also included the specialty category of the patient's first prescriber (family medicine, internal medicine, nurse practitioner, or physician assistant), which may be related to the urgency under which the first appointment was scheduled (e.g., a more acute patient may schedule a last-minute appointment with a covering nurse practitioner or physician assistant).

Second, patient characteristics and clinical factors may have influenced both the patient's access to and preference for an alternate primary care clinician, as well as their likelihood of continuing long-term opioid use (Green 2012, Scholl et al. 2019). As such, we included the following patient characteristics: age, sex, payer type, disability status (defined as patients under 65 qualified for a Medicare plan). Condition-related controls included the ICD diagnosis chapter common to the initial and follow-up appointments of the episode, as well as a set of binary variables identifying chronic conditions within the patient's medical history: asthma, coronary artery disease, congestive heart failure, chronic obstructive pulmonary disease, depression, diabetes, and hypertension.

Last, we controlled for the context in which the initial opioid prescription was made. As the study included over five years of observations for patients located across the United States, macro-level changes to primary care scheduling practices or opioid management may have occurred during that time (Dowell et al. 2017, Grosser et al. 2017). For example, patients have become more aware of the risks associated with opioids throughout the study period, e.g., driven by media attention. As such, a time covariate that counts the months since the beginning of the study. We also linked the patient's address information to the annual state-level opioid prescription rate to account for the opioid prescribing culture in the patient's state at the start of their episode (Centers for Disease Control and Prevention 2018).

Within this set of context-related controls, we also adjusted for geographical disparities in opioid prescription and overdose rates by controlling for the patient's geographical census region as well as a nine-level classification of the degree of urbanization and proximity to a metropolitan area for the patient's home address (Cromartie 2013). In addition, we controlled for the opioid-prescribing practices of the initial primary care provider. For this covariate, we calculated the rate at which the prescribing clinician's other patients continued long-term opioid use 12 months after opioid initiation. Supplement Methods S1-S2 contain further details on definitions and calculations of the control variables.

\footnotetext{
${ }^{2}$ To estimate the average daily opioid strength, the total MME of the initial prescription was divided by the days supplied reported in the pharmaceutical claims (Shah et al. 2017). If multiple prescriptions were filled on the same day, we combined the total MME and divided by the longest period of days supplied.
} 


\subsection{Identifying the Regular Primary Care Provider}

To investigate the different discordant care pathways, we used up to two years of a patient's medical history prior to opioid initiation to infer the patient's regular primary care provider. This regular provider was identified as the provider who saw the patient most frequently; in case of a tie, the regular provider was assigned based on which provider saw the patient most recently. As detailed in Supplement Methods S2 and S8, we could identify a regular provider for 10,465 of the 11,340 episodes. We then created an alternate independent variable with the following four levels: (1) provider concordance $(\mathrm{N}=8,129)$, (2) patient saw their regular provider for the initial prescribing appointment and had a follow-up with an alternate clinician $(\mathrm{N}=1,123)$, (3) patient saw an alternative clinician for their initial prescribing appointment and followed up with their regular provider ( $N=934)$, and (4) patient experienced provider discordance, but neither clinician involved was identified as the patient's regular clinician $(\mathrm{N}=1,154)$

\section{STATISTICAL ANALYSIS AND RESULTS}

\subsection{Testing Hypothesis 1: The Main Effect}

We first tested Hypothesis 1 using a standard logistic model on the 11,340-episode sample. The full model output and a description of the estimation procedure are provided in Supplement Methods S4. As summarized in Table 1 , the estimation results for the logistic models identify a large and statistically significant effect of provider discordance on long-term opioid use.

\begin{tabular}{|llllll|}
\hline Control Structure & None & $\begin{array}{l}\text { Initial } \\
\text { Prescription } \\
\left(O_{i}\right)\end{array}$ & $\begin{array}{l}\text { + Patient } \\
\text { Character- } \\
\text { istics }\left(P_{i}\right)\end{array}$ & $\begin{array}{l}+ \text { Patient } \\
\text { Conditions } \\
\left(C_{i}\right)\end{array}$ & $\begin{array}{l}\text { + Context } \\
\left(T_{i}\right)\end{array}$ \\
\hline Initial Prescription $\left(O_{i}\right)$ & No & Yes & Yes & Yes & Yes \\
Patient Characteristics $\left(P_{i}\right)$ & No & No & Yes & Yes & Yes \\
Patient Condition $\left(C_{i}\right)$ & No & No & No & Yes & Yes \\
Context $\left(T_{i}\right)$ & No & No & No & No & Yes \\
\hline
\end{tabular}

Adjusted Odds Ratio of Provider Discordance on Long-Term Opioid Use: Days Supplied Measurement

\begin{tabular}{llllll}
\hline Adjusted Odds Ratio & 0.51 & 0.66 & 0.72 & 0.70 & 0.69 \\
95\% Confidence Interval & $(0.43-0.60)$ & $(0.55-0.78)$ & $(0.60-0.85)$ & $(0.59-0.84)$ & $(0.57-0.82)$ \\
P-Value & $<0.001^{* * *}$ & $<0.001^{* * *}$ & $<0.001^{* * *}$ & $<0.001^{* * *}$ & $<0.001^{* * *}$ \\
AUC $^{+}$ & 0.56 & 0.78 & 0.82 & 0.83 & 0.84 \\
\hline Adjused & &
\end{tabular}

Adjusted Odds Ratio of Provider Discordance on Long-Term Opioid Use: Daily Strength Measurement

\begin{tabular}{llllll|}
\hline Adjusted Odds Ratio & 0.47 & 0.61 & 0.66 & 0.65 & 0.63 \\
95\% Confidence Interval & $(0.38-0.58)$ & $(0.49-0.77)$ & $(0.53-0.83)$ & $(0.51-0.82)$ & $(0.50-0.80)$ \\
P-Value & $<0.001^{* * *}$ & $<0.001^{* * *}$ & $<0.001^{* * *}$ & $<0.001^{* * *}$ & $<0.001^{* * *}$ \\
AUC $^{+}$ & 0.56 & 0.80 & 0.84 & 0.85 & 0.86 \\
\hline
\end{tabular}

${ }^{*} \mathrm{p}<0.05,{ }^{* *} \mathrm{p}<0.01,{ }^{* * *} \mathrm{p}<0.001 ; \mathrm{AUC}^{+}=$Area under the receiver operating characteristics (ROC) curve

Table 1. Logistic models. The models consistently estimate a significant effect of provider discordance on longterm opioid use at the $0.1 \%$ level, suggesting that the effect of provider discordance was not driven by model specification. The largest gain in fit (measured by $A U C$ ) comes from the initial prescription controls; additional patient characteristics, condition, and context controls make little difference to model fit or estimates. 
After accounting for all initial prescription, patient, condition, and context covariates in the episode-level logistic model, we found that patients who experienced provider discordance were less likely to become long-term opioid users compared to those who returned to the initial prescribing clinician for the follow-up appointment (adjusted odds ratio of 0.69 [ $95 \% \mathrm{CI}:(0.57,0.82)]$ using the days supplied measure and 0.63 [95\% CI: $(0.50,0.80)]$ using the alternative daily strength outcome measure). These findings support Hypothesis 1.

\subsection{Investigating Endogeneity Bias Within the Main Effect}

Although results of the controlled logistic models above are in line with Hypothesis 1, the data are retrospective, and patients were not assigned to a control group (provider concordance) or treatment group (provider discordance) at random. Consequently, coefficients may be biased by the presence of unobserved confounders that simultaneously affected the likelihood of a patient experiencing provider discordance and their likelihood of becoming a long-term opioid user. We therefore introduced two additional analysis techniques to identify and correct for endogeneity biases: (1) an instrumental variable method and (2) a propensity-score matching approach called the minimum-bias estimator.

\subsubsection{Instrumental Variable Method}

When correctly specified, the instrumental variable (IV) approach addresses concerns about self-selection bias in the model by effectively randomizing patient assignment into the exposure group to balance differences in both observed and unobserved confounders (Clarke and Windmeijer 2012, Heckman 1979). This technique makes it possible to accurately estimate causal treatment effects despite the lack of randomization in our study design (Wooldridge 2015 p. 594).

The IV approach typically requires the inclusion of an IV: a variable that should be predictive of the treatment (i.e., relevant) but have no direct impact on the outcome, nor be correlated with omitted variables that affect the outcome (i.e., valid). We chose the IV as the rate at which the prescribing clinician's other patients saw a different clinician for their follow-up appointment. Specifically, for each initial prescriber, we identified primary care appointments in the 12 months prior to the start of the episode where the patients in those appointments had a follow-up primary care visit within 30 days. The prescribing clinician's switching rate was defined as the proportion of appointments where their past patients saw a different clinician for their follow-up appointment.

If the prescribing clinician's other patients were frequently changing clinicians for their follow-up appointment (for any reason, e.g., scheduling availability of the provider), we expected the focal patient of the episode also to be more likely to experience provider discordance (i.e., relevant). However, because the IV is constructed using patients other than the focal patient, we have no reason to expect that the switching rate of these other patients should directly impact the focal patient's likelihood of becoming a long-term opioid user (i.e., valid).

That said, the IV may be invalidated if other, unobserved, factors (such as a patient's motivation to seek out nonopioid treatment) are correlated with both the IV and the likelihood of long-term opioid use. The extensive set of covariates - including the long-term opioid use rate for other opioid initiates at the same initial prescriber reduces the likelihood that this will be the case. Further exploration of the IV relevance and validity is presented in Supplement Methods S5. 
Using this IV, we estimated a patient's selection for treatment and outcome simultaneously in a recursive bivariate model:

$$
\begin{aligned}
& \text { Treatment Equation: } x_{i}^{*}=\alpha+\beta_{1} z_{i}^{\prime}+b_{1} V_{i}^{\prime}+\gamma_{1} O_{i}^{\prime}+\delta_{1} P_{i}^{\prime}+\lambda_{1} C_{i}^{\prime}+\theta_{1} T_{i}^{\prime}+\varepsilon_{2 i}, \\
& \text { Outcome Equation: } y_{i}^{*}=\alpha+\beta_{2} x_{i}+b_{2} V_{i}^{\prime}+\gamma_{2} O_{i}^{\prime}+\delta_{2} P_{i}^{\prime}+\lambda_{2} C_{i}^{\prime}+\theta_{2} T_{i}^{\prime}+\varepsilon_{1 i}, \\
& x_{i}=\mathbb{I}\left[x_{i}^{*}>0\right], y_{i}=\mathbb{I}\left[y_{i}^{*}>0\right],
\end{aligned}
$$

where $x_{i}^{*}$ and $y_{i}^{*}$ are latent variables indicating provider discordance and long-term opioid usage, respectively. The vectors $O_{i}, P_{i}, C_{i}$, and $T_{i}$ contain the set of all prescription, patient, condition, and context covariates, respectively, $\mathbb{I}[\cdot]$ is the indicator function, $z_{i}^{\prime}$ contains the IV and $V_{i}^{\prime}$ contains an additional binary IV control that equals 1 when fewer than ten observations are available to calculate the IV and 0 otherwise. The error terms $\varepsilon_{1 i}$ and $\varepsilon_{2 i}$ are jointly distributed, with the estimated correlation capturing the aggregate effect of all omitted variables that affect selection into both the treatment and outcome.

To minimize misspecification error, we imposed no prior restriction on the distributional form of $\left(\varepsilon_{1 i}, \varepsilon_{2 i}\right)$. We tested a variety of link functions (probit, logit, complementary log-log [cloglog]) and joint error distributions (modeled as copulas, such as Normal, Clayton, and Gumbel) to allow for either bivariate normal or non-normal dependencies between the treatment and outcome equations. The Supplement Methods S6 contains more details on the methodology and results from the full set of model specifications.

In the selection equation, the IV was highly predictive of provider discordance: a coefficient of 0.23 (95\% CI: [0.22-0.25], $\mathrm{p}<0.001$ ) was estimated for a $10 \%$ change in the IV. The bivariate models indicated an insignificant or small positive selection bias across a range of model specifications, as evidenced by the respective Kendall tau parameters. The best-fit recursive bivariate model with probit-logit marginals and a Clayton copula yielded an adjusted odds ratio of provider discordance of 0.46 (95\% CI: $[0.30,0.70])$ using the days supplied outcome measure and $0.42(95 \% \mathrm{CI}:[0.24,0.72])$ using the daily strength outcome measure. As such, the results from the logistic model appear conservative.

\subsubsection{Minimum Bias Estimator}

Despite the range of sensitivity analyses we performed on the IV model (see Supplement Methods S5-S6) to validate the IV and corresponding results, there may still be concerns about the validity of the instrument. We therefore leveraged a second approach to address endogeneity concerns based on propensity-score matching methods. We matched patients in the control group (concordance) in a 1:1 ratio with patients in the treatment group (discordance) using nearest neighbor matching, with the condition that the closest propensity score can be no greater than 0.2 standard deviations away from the switchers' propensity score. This condition has been shown to reduce more than $90 \%$ of the bias due to observable differences ( $\mathrm{Gu}$ and Rosenbaum 1993) as well alleviate some of the impact of unobserved bias (Rosenbaum 2005).

We then followed a technique detailed by Millimet and Tchernis (2013, p. 983) that uses the propensity scores from matching to estimate a minimum-biased estimator (MBE). The MBE aims to minimize the potential impact of omitted variable bias by restricting the sample to matched cases with propensity scores within a defined interval around 0.5 , the propensity score which most closely mimics a coin-flip or random assignment to the 
treatment and control groups. By estimating the logistic model on increasingly restricted subsamples (for example, limiting to propensity scores between 0.10 and 0.90 , then 0.25 to 0.75 , etc.), the method will allow us to detect the direction of endogeneity and reduce bias in the estimated effect size.

As shown in Table 2, the adjusted odds ratios get increasingly smaller as the range of propensity scores narrow. When we reach the narrowest propensity-score range ([0.33, 0.67], as recommended by Black and Smith (2004)) the estimated size of the effect of provider discordance on long-term opioid use increases to 0.55 (95\% CI: [0.38$0.79])$ and 0.45 (95\% CI: [0.28-0.73]) for the days supplied and daily strength measures, respectively. ${ }^{3}$ These estimates of the effect size using the MBE approach are similar to those observed using the IV approach.

\begin{tabular}{|l|l|l|l|l|}
\cline { 2 - 5 } \multicolumn{1}{c|}{} & \multicolumn{2}{l|}{ Days Supplied Measurement } & \multicolumn{2}{l|}{ Daily Strength Measurement } \\
\hline $\begin{array}{l}\text { Adjusted Odds Ratio on Long-Term } \\
\text { Opioid Use (95\% Confidence } \\
\text { Interval) }\end{array}$ & $\begin{array}{l}\text { Adjusted Odds } \\
\text { Ratio }\end{array}$ & P-value & $\begin{array}{l}\text { Adjusted Odds } \\
\text { Ratio }\end{array}$ & P-value \\
\hline Full sample (N=11,340) & $0.69(0.57-0.82)$ & $<0.001^{* * *}$ & $0.62(0.50-0.80)$ & $<0.001^{* * *}$ \\
\hline Propensity matched sample (N=6,084) & $0.75(0.62-0.91)$ & $0.003^{* *}$ & $0.62(0.48-0.79)$ & $<0.001^{* * *}$ \\
\hline $\begin{array}{l}\text { Propensity matched sample with } \\
\text { scores 0.10-0.90 (N=5,966) }\end{array}$ & $0.72(0.59-0.88)$ & $0.001^{* *}$ & $0.59(0.46-0.76)$ & $<0.001^{* * *}$ \\
\hline $\begin{array}{l}\text { Propensity matched sample with } \\
\text { scores 0.25-0.75 (N=4,113) }\end{array}$ & $0.65(0.49-0.87)$ & $0.003^{* *}$ & $0.45(0.32-0.65)$ & $<0.001^{* * *}$ \\
\hline $\begin{array}{l}\text { Propensity matched sample with } \\
\text { scores 0.33-0.67 (N=2,745) }\end{array}$ & $0.55(0.38-0.79)$ & $0.001^{* *}$ & $0.45(0.28-0.73)$ & $0.001^{* *}$ \\
\hline
\end{tabular}

$\wedge \mathrm{p}<0.10,{ }^{*} \mathrm{p}<0.05,{ }^{*} \mathrm{p}<0.01, * * * \mathrm{p}<0.001$

Table 2. Estimated effect of provider discordance using the Minimum Bias Estimator method. The propensity-matched sample is reduced to observations with scores closer to 0.5 to correct for unobserved confounders. The estimates of the effect of provider discordance on long-term opioid use become more negative as endogeneity bias is increasingly addressed.

In summary, the IV and MBE methods consistently suggest that unobserved factors, such as self-selection, rendered the group of patients who experienced discordance more likely to become long-term opioid users. In other words, those patients who chose not to change provider or who were unable to do so (i.e., from limited provider access in their region) would have benefitted even more from discordant care than those patients in our sample who already received the treatment. In conjunction with additional analyses presented in Supplement Part VI, all evidence indicates that the logistic model estimates underestimate the beneficial effect of provider discordance on long-term opioid use and support Hypothesis 1.

\subsection{Testing Hypothesis 2: A Mechanism}

Hypotheses $2 \mathrm{a}$ and $2 \mathrm{~b}$ investigate a potential mechanism (a change in short-term opioid usage) that could explain the relationship between provider discordance and long-term opioid use. To test the hypotheses, we separately estimated the following equations:

$$
\begin{gathered}
m_{i}^{*}=\mu+\eta x_{i}+O_{i}^{\prime} v+P_{i}^{\prime} \xi+C_{i}^{\prime} \varphi+T_{i}^{\prime} \psi+v_{i}, m_{i}=\mathbb{I}\left[m_{i}^{*}>0\right] \\
y_{i}^{*}=\alpha+\omega m_{i}+O_{i}^{\prime} \gamma+P_{i}^{\prime} \delta+C_{i}^{\prime} \lambda+T_{i}^{\prime} \theta+\varepsilon_{i}, y_{i}=\mathbb{I}\left[y_{i}^{*}>0\right]
\end{gathered}
$$

\footnotetext{
${ }^{3}$ Note that the p-values may be less significant as the effects are estimated on a smaller sample.
} 
where $m_{i}^{*}$ captures whether the patient filled an opioid prescription after their follow-up appointment, and both $\nu$ and $\varepsilon$ are error terms following the standard logistic distribution. To remain consistent with the 14-day time window used when matching the initial prescription with the prescribing appointment, this subsequent opioid prescription must have been filled within 14 days of that follow-up appointment. The coefficient $\eta$ in Equation [3] captures the extent to which patients who change clinicians for their follow-up appointment are more likely to fill a subsequent opioid prescription (Hypothesis 2a). Meanwhile, $\omega$ in Equation [4] estimates the association between filling a subsequent prescription and becoming a long-term opioid user (Hypothesis $2 b$ ).

The model estimates support Hypotheses $2 \mathrm{a}$ and $2 \mathrm{~b}$. Patients who experienced provider discordance were less likely to fill an opioid prescription in the subsequent 14-day period (adjusted odds ratio 0.79 [95\% CI: 0.71 , $0.88]$ ). Furthermore, patients who filled an opioid prescription within 14 days after the follow-up appointment were much more likely than those who did not to become long-term users (adjusted odds ratio 6.31 [95\% CI: 5.41, 7.36] using the days supplied outcome measure, and 5.34 [95\% CI: 4.40, 6.49] using the daily strength outcome measure). While we do not claim causal identification using these models, these correlational results identify a potential mechanism underlying the effect that provider discordance has on long-term opioid use.

\subsection{Testing Hypothesis 3: Provider Discordance Pathways}

To test the consistency of the provider discordance effect, we examined whether the strength of the effect differed depending on whether and when the patient saw their regular provider. In the fully controlled logistic models, we replaced $x_{i}$ with the categorical variable $X_{i}$ which has four levels corresponding to each of the pathways described in Section 3.6. We also added a binary variable $r_{i}$ that specified whether the patient saw their regular provider for either the first or second appointment $\left(r_{i}=0\right.$ if neither the first nor the second appointment was with the regular provider). This ensured that any advantage associated with a patient seeing their regular provider during the observed care episode was already accounted for in the models before testing for the potential additional benefit of discordant care.

$$
y_{i}^{*}=\alpha+\beta X_{i}+\sigma r_{i}+\gamma O_{i}^{\prime}+\delta P_{i}^{\prime}+\lambda C_{i}^{\prime}+\theta T_{i}^{\prime}+\varepsilon_{i}, y_{i}=\mathbb{I}\left[y_{i}^{*}>0\right] .
$$

As displayed in Table 3 (and further detailed in Supplement Methods S8), the effect of provider discordance appears strongest for the set of patients who started with a non-regular provider for their first visit then switched to their regular PCP (adjusted odds ratio 0.58 [95\% CI: (0.40-0.84)] and 0.51 [95\% CI: (0.31-0.84)] for the days supplied and daily strength measurements, respectively). However, even for those patients who start with their regular provider and then switch to an alternative clinician, we find that discordance reduces their likelihood of becoming long-term opioid users.

We ran a series of hypothesis tests to determine whether the discordant care effect sizes are statistically different depending on the discordance pathway. The corresponding p-values (all greater than 0.31 using the chi-squared test) do not allow us to reject the hypothesis that the effect sizes are the same. While the sample size may limit the power to identify significant differences in effect sizes, the above results indicate that all types of patient pathways (even those where the patient is initially prescribed by their regular PCP) benefit from provider discordance at the beginning of the opioid episode. As such, we do not find evidence in support of Hypothesis 3. 


\begin{tabular}{|c|c|c|c|c|}
\hline \multirow[b]{2}{*}{ Effect on Long-Term Opioid Use } & \multicolumn{2}{|c|}{$\begin{array}{l}\text { Days Supplied } \\
\text { Measurement }\end{array}$} & \multicolumn{2}{|c|}{$\begin{array}{l}\text { Daily Strength } \\
\text { Measurement }\end{array}$} \\
\hline & $\begin{array}{l}\text { Adjusted Odds } \\
\text { Ratio (95\% CI) }\end{array}$ & P-value & $\begin{array}{l}\text { Adjusted Odds } \\
\text { Ratio (95\% CI) } \\
\end{array}$ & P-value \\
\hline $\begin{array}{l}\text { Discordance pathway 1: regular provider to } \\
\text { alternate provider }\end{array}$ & $0.73(0.56-0.94)$ & $0.014 *$ & $0.68(0.49-0.95)$ & $0.024 *$ \\
\hline $\begin{array}{l}\text { Discordance pathway 2: alternate provider to } \\
\text { regular provider }\end{array}$ & $0.58(0.40-0.84)$ & $0.004 * *$ & $0.51(0.31-0.84)$ & $0.008^{* *}$ \\
\hline $\begin{array}{l}\text { Discordance pathway 3: alternate provider to } \\
\text { alternate provider }\end{array}$ & $0.72(0.53-0.97)$ & $0.034 *$ & $0.65(0.44-0.97)$ & $0.036^{*}$ \\
\hline
\end{tabular}

Table 3. Effect of provider discordance within different pathways. Pathways describe whether and when the patient was seen by their regular (most frequently visited) primary care provider, during the initial prescribing appointment or follow-up appointment.

\subsection{Alternative Explanations and Robustness Tests}

One alternative explanation for our findings is that provider discordance is a proxy for the acuity of pain onset. High acuity patients may be more likely to receive an opioid script from a covering clinician (due to unavailability at short notice of their preferred provider) and then return to their regular provider for follow-up. If this is the case, these patients may be simultaneously more likely to experience discordance between the initial providers and less likely to become long-term opioid users (as their condition is more acute than chronic).

This potential omitted variable has been addressed in part by both the IV and MBE approaches, as they are designed to estimate the causal effect of provider discordance after adjusting for potential confounds such as acuity (see Supplement Methods S5-S7 for further discussion). In addition, the pathway analysis described in Section 4.4 indicates that all provider discordance pathways lead to reduced long-term opioid use rates, i.e., the effect we find does not appear to be explained exclusively by patients switching from a covering clinician to their regular clinician (the pathway expected to occur more likely amongst acute patients).

Despite these analyses, we acknowledge that unobserved factors that may still bias the results. For example, certain providers may be more available than others for more urgent appointments, and so may have a higher proportion of patients with acute pain onsets. To account for this and for other forms of provider-level heterogeneity, we included fixed and random effects at the initial prescriber-level in Supplement Methods S11. In Supplement Methods S12, we explored whether the observed effect of provider discordance differed depending on whether opioid initiates were being treated for a new (likely more acute pain) or a potentially preexisting condition. In Supplement Methods S13, we included additional controls (available for $80.2 \%$ of patient episodes) that estimated the patient's socioeconomic status (differences which have been associated with variation in opioid use) and the estimated average patient knowledge of opioid risk, as measured by changes to the media attention and interest in the opioid epidemic across the study period. Other sensitivity analyses include relaxing the assumption that provider discordance must occur during the first two appointments of the patient's opioid journey (Supplement Methods S15), and measuring the impact of provider discordance on opioid use at different points throughout the first year, e.g., 6 months, 9 months, etc. (Supplement Methods S16). All estimates were consistent with the main results. 


\section{DISCUSSION AND PRACTICAL IMPLICATIONS}

\subsection{Discussion and Policy Recommendations}

As opioid overdoses tend to occur with patients who are already in a pattern of chronic opioid use, many intervention strategies have aptly approached the opioid epidemic from the perspective of clinical management of ongoing dependence (Grosser et al. 2017, Hallvik et al. 2018, Pitt et al. 2018). However, our analysis suggests that operational interventions during the early phases of opioid use may also be extremely valuable. For opioidnaïve patients initially managed with an opioid treatment in the primary care setting, this research identified that clinician discordance can significantly disrupt the path to long-term opioid use.

A logistic regression model estimated that the likelihood of long-term opioid use at 360 days is $31 \%$ lower ( $95 \%$ CI: $[18 \%, 43 \%])$ for patients who followed up with an alternate primary care clinician, controlling for the strength and days supplied of the initial opioid prescription (known to be associated with risk of long-term opioid use and overdose) as well as a range of patient, condition, and context factors. Robustness tests including an IV analysis and propensity-score matching (utilizing the MBE approach) suggest that this estimate is, if anything, a conservative assessment of the true causal effect. While multiple potential mechanisms may explain the difference in long-term opioid use, one identified explanatory factor is that patients who experienced provider discordance were also $21 \%$ (95\% CI: [12\%, 29\%]) less likely to fill a subsequent opioid prescription after the follow-up appointment. This decrease can have a large long-term impact, as filling an opioid prescription after the follow-up appointment was associated with a 6.31 [95\% CI: 5.41, 7.36] times higher likelihood of continuing long-term opioid use.

We also performed a pathway analysis to assess whether the effects of provider discordance remained significant for patients whose initial prescriber was their regular provider. While there was evidence of a stronger effect on patients whose initial prescriber was not their non-regular clinician - with long-term opioid use at 360 days for such patients reduced by $42 \%$ (95\% CI: $[16 \%, 60 \%])$ - the difference compared to other pathways was not statistically significant. This indicates that the benefits of provider discordance are pervasive and that, on average, patients benefit from seeing a different clinician regardless of who they saw for the first or follow-up appointments.

While it is critical for the results of this study to be corroborated through other data sets and study designs, there are multiple potential policy changes that could help facilitate provider discordance within the early stages of opioid use. As an example, approximately $85 \%$ of primary care physicians practice in an office with at least one other physician (Liaw et al. 2016). If a patient is flagged in the medical record system upon receiving a new opioid prescription, and the patient subsequently contacts the practice to schedule a follow-up appointment, then the office manager could schedule this appointment with a clinician other than the initial prescriber. ${ }^{4}$ Without posing significant additional overhead or risking patient loss for practices involved, such a policy could impact a large portion of new opioid initiates in the primary care setting. As an operational process change, this intervention also circumvents the need for clinician education and other resource-intensive efforts to elicit

\footnotetext{
${ }^{4}$ As shown in Supplement S10, we did not observe any significant difference in the effect of provider discordance when the two clinicians shared an office versus when the two clinicians operated out of different offices.
} 
longstanding behavior change for individual providers (Morris et al. 2011). In a new era of opioid management where tools such as prescription drug monitoring programs are becoming increasingly available for clinicians to see a broader picture of the patient's history and opioid-use patterns, the medical community is well poised to incorporate safeguarding measures focused on the early stages of opioid use.

\subsection{Limitations}

While these results provide promising evidence that the design of care management shortly after opioid initiation may significantly disrupt the pathway to long-term opioid use, the implications are not without limitations. First, it must be emphasized that this research does not claim that all opioid use is unnecessary or that conservative, alternative treatments are always more appropriate to manage ongoing pain; while aggressive opioid prescribing may induce significant clinical harm without providing meaningful pain improvement, some patients and conditions may respond well to pain management through opioids (Glod 2017). There is also a possibility that provider discordance shortly after opioid initiation could negatively affect other aspects of care quality. ${ }^{5}$

Second, this study is focused on new opioid initiates who return for a follow-up appointment in the primary care setting. As such, the findings cannot necessarily be extended to all new opioid users - for instance, to patients who require a one-off prescription or who have higher acuity pain that requires them to receive their first prescription in an emergency or urgent care setting. While our analyses show an aggregate net positive effect of introducing provider discordance within the primary care setting (see Supplement Methods S14), further analyses should investigate the impact of potential policy interventions on specific at-risk populations.

Third, although there are plausible explanations as to why provider discordance might be effective in this context (including an enlarged information base and correction to confirmation bias and anchoring), this study does not identify the precise behavioral mechanism behind the observed effect. Developing a clearer understanding of why this effect exists and whether the impact would be observed in other (i.e., non-opioid related) contexts could help researchers design and target potential interventions effectively.

Last, while we have taken various steps in our paper to address the issue of causality (e.g., by including an extensive set and combinations of controls in sensitivity analyses and employing IV and MBE methods), as is the case with all observational studies we cannot fully rule out the possibility of omitted variable bias. Although retrospective cohort studies are the norm in opioid research (Longhurst et al. 2014) and we recommend that similar analyses be replicated with other datasets, the only way to definitively confirm our findings is through a randomized control trial. The size and consistency of our findings across models suggest that such trials and corresponding investment into designing and testing clinical interventions may be warranted.

\subsection{Conclusion}

High-quality medical decision-making is at the heart of good medicine, yet it cannot be taken for granted. This study demonstrates that systematic operational changes in the primary care setting following opioid initiation may be a promising target to reduce the influx of patients afflicted by the opioid epidemic. We advocate for future

\footnotetext{
${ }^{5}$ In Supplement Methods S17, we investigate whether provider discordance shortly after opioid initiation is associated with an increase in emergency room usage (a quality measure that indicates poor pain management). While we recommend further analyses to explore this possibility of negative externalities, we did not identify any effect.
} 
research to identify potential interventions early in the process of opioid management that can safely and effectively lower rates of long-term opioid use.

\section{REFERENCES}

Ahuja V, Alvarez C, Staats BR (2020) Maintaining Continuity in Service: An Empirical Examination of Primary Care Physicians. Manuf. Serv. Oper. Manag.

American Medical Association (2020) Issue brief: Reports of Increases in opioid-related overdose during COVID pandemic

Ariely D, Loewenstein G, Prelec D (2003) "Coherent arbitrariness": Stable demand curves without stable preferences. Q. J. Econ. 118(1):73-105.

Atlas S J, Grant R W, Ferris T G, Chang Y, Barry M J, Atlas Steven J, Grant Richard W, Ferris Timothy G, Chang Yuchiao, Barry Michael J (2009) Patient-physician connectedness and quality of primary care. Ann. Intern. Med. 150(5):325-335.

Ayer T, Alagoz O, Stout NK (2012) OR forum-A POMDP approach to personalize mammography screening decisions. Oper. Res. 60(5):1019-1034.

Barnett M, Olenski A, Jena A (2017) Opioid-Prescribing Patterns of Emergency Physicians and Risk of LongTerm Use. N. Engl. J. Med. 376(7):663-673.

Black DA, Smith JA (2004) How robust is the evidence on the effects of college quality? Evidence from matching. J. Econom. 121(1-2):99-124.

Brooks AW, Gino F, Schweitzer ME (2015) Smart people ask for (My) advice: Seeking advice boosts perceptions of competence. Manage. Sci. 61(6):1421-1435.

Centers for Disease Control and Prevention (2018) U.S. Opioid Prescribing Rate Maps. U.S. Dep. Heal. Hum. Serv. Opioid Overdose. Retrieved (July 25, 2019), https://www.cdc.gov/drugoverdose/maps/rxratemaps.html.

Centers for Medicare and Medicaid Services (2018) Opioid Oral Morphine Milligram Equivalent (MME) Conversion Factors. Prescr. Drug Cover.:1-2. Retrieved https://www.cms.gov/Medicare/PrescriptionDrug-Coverage/PrescriptionDrugCovContra/.

Chan CW, Farias VF, Bambos N, Escobar GJ (2012) Optimizing Intensive Care Unit Discharge Decisions with Patient Readmissions. Oper. Res. 60(6):1323-1341.

Chan CW, Green L V., Lekwijit S, Lu L, Escobar G (2019) Assessing the impact of service level when customer needs are uncertain: An empirical investigation of hospital step-down units. Manage. Sci. 65(2):751-775.

Chiu AS, Jean RA, Hoag JR, Freedman-Weiss M, Healy JM, Pei KY (2018) Association of Lowering Default Pill Counts in Electronic Medical Record Systems with Postoperative Opioid Prescribing. JAMA Surg. 153(11):1012-1019.

Christensen CM, Grossman JH, Hwang J (2009) The Innovator's Prescription: A Disruptive Solution for Health Care (McGraw-Hill).

Clark JR, Huckman RS, Staats BR (2013) Learning from customers: Individual and organizational effects in outsourced radiological services. Organ. Sci. 24(5):1539-1557.

Clarke PS, Windmeijer F (2012) Instrumental variable estimators for binary outcomes. J. Am. Stat. Assoc. 
107(500):1638-1652.

Compton WM, Jones CM, Baldwin GT (2016) Relationship between Nonmedical Prescription-Opioid Use and Heroin Use. N. Engl. J. Med. 374(2):154-163.

Cromartie J (2013) Rural-Urban Continuum Codes. US Dep. Agric. Econ. Res. Serv. Retrieved (November 19, 2019), https://www.ers.usda.gov/data-products/rural-urban-continuum-codes.aspx.

Deyo RA, Hallvik SE, Hildebran C, Marino M, Dexter E, Irvine JM, O’Kane N, et al. (2017) Association Between Initial Opioid Prescribing Patterns and Subsequent Long-Term Use Among Opioid-Naïve Patients: A Statewide Retrospective Cohort Study. J. Gen. Intern. Med. 32(1):21-27.

Dickerson JP, Procaccia AD, Sandholm T (2019) Failure-aware kidney exchange. Manage. Sci. 65(4):1768-1791.

Dowell D, Arias E, Kochanek K, Anderson R, Guy GPJ, Losby J, Baldwin G (2017) Contribution of OpioidInvolved Poisoning to the Change in Life Expectancy in the United States, 2000-2015. JAMA 318(11):10651067.

Dowell D, Haegerich TM, Chou R (2016) CDC Guideline for Prescribing Opioids for Chronic Pain - United States, 2016. JAMA 315(15):1624-1645.

Farley KX, Anastasio AT, Kumar A, Premkumar A, Gottschalk MB, Xerogeanes J (2019) Association Between Quantity of Opioids Prescribed After Surgery or Preoperative Opioid Use Education With Opioid Consumption. JAMA 321(24):2465-2466.

Freeman M, Robinson S, Scholtes S (2020) Gatekeeping, Fast and Slow: An Empirical Study of Referral Errors in the Emergency Department. Manage. Sci. Forthcomin.

Freeman M, Savva N, Scholtesa S (2017) Gatekeepers at work: An empirical analysis of a maternity unit. Manage. Sci. 63(10):3147-3167.

Gino F, Pisano G (2008) Toward a theory of behavioral operations. Manuf. Serv. Oper. Manag. 10(4):676-691.

Glod SA (2017) The other victims of the opioid epidemic. N. Engl. J. Med. 376(22):2101-2102.

Green L V. (2012) The vital role of operations analysis in improving healthcare delivery. Manuf. Serv. Oper. Manag. 14(4):488-494.

Grosser BT, Woolf CJ, Fitzgerald GA (2017) Time for nonaddictive relief of pain. Science (80-. ). 355(6329):1026-1027.

Gu XS, Rosenbaum PR (1993) Comparison of Multivariate Matching Methods: Structures, Distances, and Algorithms. J. Comput. Graph. Stat. 2(4):405-420.

Hallvik S, Geissert P, Wakeland W, Hildebran C, Carson J, O'Kane N, Deyo R (2018) Opioid-Prescribing Continuity and Risky Opioid Prescriptions. Ann. Fam. Med. 16(5):440-442.

Heckman JJ (1979) Sample Selection Bias as a Specification Error. Econometrica 47(1):153-161.

Holland KM, Jones C, Vivolo-Kantor AM, Idaikkadar N, Zwald M, Hoots B, Yard E, et al. (2021) Trends in US Emergency Department Visits for Mental Health, Overdose, and Violence Outcomes Before and During the COVID-19 Pandemic. JAMA Psychiatry 30341:1-8.

Hoopes M, Angier H, Raynor LA, Suchocki A, Muench J, Marino M, Rivera P, Huguet N (2018) Development of an algorithm to link electronic health record prescriptions with pharmacy dispense claims. J. Am. Med. Informatics Assoc. 25(10):1322-1330.

Hopp WJ, Li J, Wang G (2018) Big Data and the Precision Medicine Revolution. Prod. Oper. Manag. 27(9):16471664. 
Jena AB, Goldman D, Weaver L, Karaca-Mandic P (2014) Opioid prescribing by multiple providers in Medicare: Retrospective observational study of insurance claims. BMJ 348(February):1-12.

Kuntz L, Scholtes S, Sülz S (2019) Separate and concentrate: Accounting for patient complexity in general hospitals. Manage. Sci. 65(6):2482-2501.

Levy B, Paulozzi L, Mack KA, Jones CM (2015) Trends in Opioid Analgesic-Prescribing Rates by Specialty, U.S., 2007-2012. Am. J. Prev. Med. 49(3):409-413.

Liaw WR, Jetty A, Petterson SM, Peterson LE, Bazemore AW (2016) Solo and small practices: A vital, diverse part of primary care. Ann. Fam. Med. 14(1):8-15.

Longhurst CA, Harrington RA, Shah NH (2014) A "green button" for using aggregate patient data at the point of care. Health Aff. 33(7):1229-1235.

Millimet DL, Tchernis R (2013) Estimation of the treatment effects without an exclusion restriction: with an application to tha analysis of the school breakfast program. J. Appl. Econom. 28:982-1017.

Morone NE, Weiner DK (2013) Pain as the fifth vital sign: Exposing the vital need for pain education. Clin. Ther. 35(11):1728-1732.

Morris ZS, Wooding S, Grant J (2011) The answer is 17 years, what is the question: Understanding time lags in translational research. J. R. Soc. Med. 104(12):510-520.

Owen GT, Bruel BM, Schade CM, Eckmann MS, Hustak EC, Engle MP (2018) Evidence-based pain medicine for primary care physicians. Baylor Univ. Med. Cent. Proc. 31(1):37-47.

Pitt A, Humphreys K, Brandeau M (2018) Modeling Health Benefits and Harms of Public Policy Responses to the US Opioid Epidemic. Am. J. Public Health 108(10):1394-1400.

Reagans R, Argote L, Brooks D (2005) Individual experience and experience working together: Predicting learning rates from knowing who knows what and knowing how to work together. Manage. Sci. 51(6):869881 .

Rosenbaum PR (2005) Heterogeneity and causality: Unit heterogeneity and design sensitivity in observational studies. Am. Stat. 59(2):147-152.

Scholl L, Seth P, Kariisa M, Wilson N, Baldwin G, Release E (2019) Drug and Opioid-Involved Overdose Deaths — United States, 2013 - 2017. Morb. Mortal. Wkly. Rep. 67(51-52):1419-1427.

Senot C (2019) Continuity of Care and Risk of Readmission: An Investigation into the Healthcare Journey of Heart Failure Patients. Prod. Oper. Manag. 28(8):2008-2030.

Shumsky RA, Pinker EJ (2003) Gatekeepers and referrals in services. Manage. Sci. 49(7):839-856.

Siemsen E, Roth A V., Balasubramanian S, Anand G (2009) The influence of psychological safety and confidence in knowledge on employee knowledge sharing. Manuf. Serv. Oper. Manag. 11(3):429-447.

Song H, Tucker A, Graue R, Moravick S, Yang J (2019) Capacity Pooling in Hospitals: The Hidden Consequences of Off-Service Placement. Manage. Sci.:1-18.

Staats BR, Diwas SKC, Gino F (2018) Maintaining beliefs in the face of negative news: The moderating role of experience. Manage. Sci. 64(2):804-824.

Wooldridge JM (2015) Introductory Econometrics: A Modern Approach Sixth. (Australia: Cengage Learning). 